\title{
Power Distribution Planning Using Multi-Criteria Decision Making Method
}

\author{
Sandeep Chakravorty, Smarajit Ghosh
}

\begin{abstract}
Conductor layout plays a vital role in distribution planning. This paper presents an improved mathematical model to optimize the network routing problem. The model was formulated to minimize the feeder routes subject to a set of constraints. The computational results of a devised sample problem indicate that the developed optimization model is adequate for planning of distribution systems.

Here in this paper we suggest the distribution planning by the means of a hybrid model which includes Taguchi and Analytical Hierarchy Process (AHP) and Technique of Order Preference by Similarity to Ideal Solution (TOPSIS) where we take into consideration some aspects of reliability such as miles of conductor, feeder failures, customer interruption, maximum interruption, estimated relative cost. The method suggested here firstly determines the priority of these aspects of reliability and then it determines the weight of these factors and ultimately distribution planning has been carried out by Technique of Order Preference by Similarity to Ideal Solution (TOPSIS). The results of the discussed techniques will lead to lower long range distribution expenses as it will lead to optimum feeder path.
\end{abstract}

Index Terms - Power distribution planning, Taguchi technique, AHP technique, TOPSIS, optimization methods.

\section{INTRODUCTION}

Decision making features occurs in all the fields of the human activities such as scientific and technological and affects every sphere of the life. Engineering design, which entails sizing, dimensioning, and detailed element planning is also not exempt from its influence.

Normally, any engineering problem will have large number of solutions out of which some of are feasible and some are infeasible. The designer's task is to get the best solution out of the feasible solutions.

The available literature consists of work of only few researchers on the field of distribution planning. Most of them are based on mathematical programming such as transportation, transshipment algorithms [8,9], mixed integer programming [10], dynamic programming [11] etc. Unfortunately only near optimal solutions have been obtained by these mathematical programming methods because almost every method has made some approximations on the model of distribution planning, moreover these methods are often complicated and time consuming.

In the work done by K.K.Li and T.S. Chung [3] genetic

Manuscript received May 4, 2009.

Sandeep Chakravorty is with Sikkim Manipal Institute of Technology under Sikkim Manipal University, Department of Electrical \& Electronics Engineering, Maitar, Rangpo, Esat Sikkim, India.

e-mail: sandeep chakravorty@yahoo.com

Smarajit Ghosh is with the Thapar University, Department of Electrical \& Instrumentation Engineering, Patiala, Punjab-147004, India

e-mail: smarajitg@hotmail.com algorithm have been used to find the optimum location of substation to meet the load demands of 13 load points whose coordinates and MVA demands are given.

Similar work has been carried out by Belgin Turkay and Taylan Artac [1], work has also been carried out by J.F.Gomez et.al., [2].

In all the above cases planning of laying the feeders or distribution planning has been done either by man machine interface or heuristic algorithm. The papers further don't take into consideration aspects of reliability which are very much essential in power distribution factors such as line down, transformer out of service, interruption, estimated relative cost etc. In the work done S. Chakravorty et.al.,[36] distribution planning by means of Analytical Hierarchy Process have been done where some aspects of reliability such as miles of conductor, feeder failures, customer interruption, maximum interruption, estimated relative cost have been taken care of but the priority of these factors have been decided in random. No technique has been used to determine the priority of importance of these factors. In this paper a model has been proposed where a systematic solution process has been used to determine the priority of these factors then an Analytical Hierarchy Process has been applied for finding the weight of these factors and ultimately distribution planning has been carried out by Technique of Order Preference by Similarity to Ideal Solution. Thus we can claim the suggested method to be more robust and more close to practical than the one suggested by S. Chakravorty et.al.,[36].

A complete survey of the proposed techniques for the solution of the planning problem of primary distribution circuits can be found in [15] and [16]. Initially the proposed methods were mainly based upon the generation and evaluation of possible solutions, oriented to small size problems, and requiring important efforts for the production of the alternatives to be evaluated. Among these the heuristic zone valuation and the generation of service areas methods may be mentioned. They rely completely upon the experience of the planning engineer and have the disadvantage that the best alternative may not be considered.

The planning problem of primary distribution networks has been basically stated as a classical mixed integer linear programming problem, where an objective function that includes both the investment and the operation cost of the network, is minimized subject to technical constraints related with the characteristics of the electric service [17]. This formulation includes binary variables linked to the fixed costs associated to some of the decision variables as well as linear approximations to represent the variable costs.

Stated as such the classical branch and bound techniques [17] have a natural application. Although the continuous 
research and improvement of these techniques keeps them as an alternative always to be considered, the linear approximations as well as the combinatorial complexity of the problem question their application to electric systems of real dimensions due to the requirements of excessive computing resources. This is the main reason for the application of alternative approaches, such as those classified as metaheuristic methods that are able to locate good solutions with reduced computational effort.

In order to reduce the computation requirements of the branch and bound optimization technique, the identification of the set of variables that affects most of the results was proposed in order to reduce the complexity of the mathematical model [16] however a linear approximation of the variable costs is required and excessive solution times are reported as the number of binary variables increases.

Heuristic search methods have been developed [17], [18], showing faster performance than the conventional optimization techniques but with some limitations in the goodness of the solutions to the problem that are obtained.

In [20] an open loop planning procedure is proposed where an expert system based upon the use of geographical information is applied to automate the selection of the primary and secondary circuit routes. GA's have also been applied to the solution of this problem, reducing the solution time and enhancing the obtained results [15],[18],[21],[22],[23]. In [22] a solution methodology based upon a GA is proposed for the design of primary distribution circuits considering multiple system expansion stages, where a special coding procedure is proposed to reduce the number of unfeasible solutions evaluated by the algorithm. These methodologies have been improved significantly. An evolutionary programming algorithm is presented in [25], considering the uncertainties of the possible scenarios. In [16] and [24] the potential of the GA's is shown in comparison with classical optimization techniques to solve the planning problem in a very complete and detailed formulation considering the nonlinearity of the cost function, the limits of the voltage magnitudes and a term in the objective function to take into account the reliability of the system, reporting significant improvements in the solution times. An integer variable coding scheme was used to facilitate the consideration of different conductor sizes and substation sizes also new genetic operators were proposed to improve the performance of the algorithm. In [26] the approach is expanded to consider the multiple development stages as well as multiple objectives. In [31] an evolutionary approach is applied to the design of a medium voltage network using a detailed model of the network. All the suggest methods fail to take into consideration aspects of reliability. This new methodology suggests a distribution planning technique taking into consideration aspects of reliability according to the priority of them.

\section{Proposed Methodology Applied To a Case Study}

Let us have the problem discussed by S. Chakravorty et.al.,[36] where a thirteen load points are to be fed from two substations depending on the capacity and the load demands. The layout of feeders for feeding the thirteen load points depends on the following factors: a). Miles of conductor.

b). Feeder failures/year.

c). Customer interruption/year.

d). Maximum interruption/customer.

e). Estimated relative cost.

Substation location has been obtained by the use of genetic algorithm stated in [33].

The pictorial representation of the problem is as shown in fig 1.

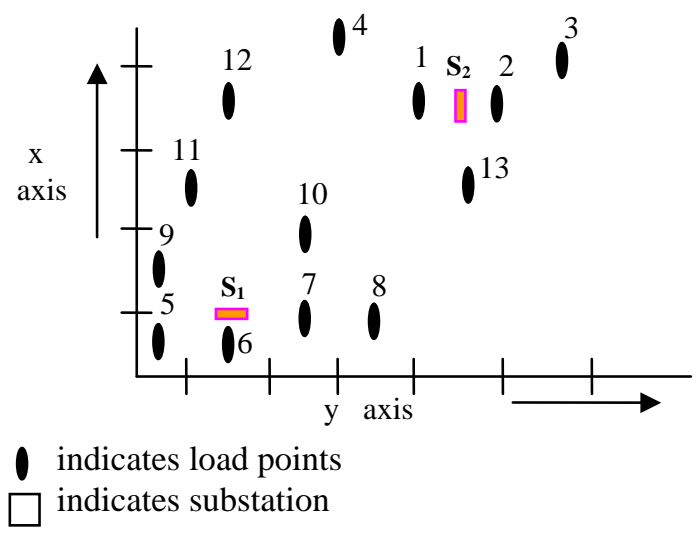

Figure 1 (Representation of the problem)

Results of genetic algorithm allots load points (7 108611

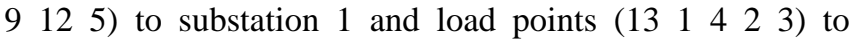
substation 2 and gives the optimum location of substation 1 as (3 2) and that of substation 2 as (9 7). Now for determining the conductor layout we apply the Multi-Criteria Decision Making methods.

Analytical Hierarchy Process is a multi-criteria decision making process that allows working with both numerical factors and those that are un-tangible and subjective. It provides a structured way to determine the weighting factors for each criterion and applies the weights to the comparison of alternatives. AHP uses pair wise comparisons of design concepts to convert a statement

( "this is vary important", "this is not so important") into a numerical value.

To determine the conductor layout following factors have been considered:

a). Miles of conductor.

b). Feeder failures/year.

c). Customer interruption/year.

d). Maximum interruption/customer.

e). Estimated relative cost.

In deciding how much more strongly does this criterion influence the outcome than the other criterion a nine point scale has been used ( 1 indicates equal importance; 3 indicates moderate importance; 5 indicates strong importance; 7 indicates very strong importance; 9 indicates extreme importance). However the priority of these factors or which of these factors are more important over others have not been considered in the work of [36]. Here before applying AHP we go for Taguchi to decide how strongly one factors influence the others. Here a three level design has been followed with 27 combinations.

\begin{tabular}{|l|l|l|l|} 
& Level 1 & Level 2 & Level 3 \\
\hline Miles of conductor & 5 & 10 & 20 \\
\hline
\end{tabular}




\begin{tabular}{|l|l|l|l|}
\hline Feeder failures/yr & 0 & 1 & 2 \\
\hline $\begin{array}{l}\text { Customer } \\
\text { interruption/year }\end{array}$ & 100 & 200 & 300 \\
\hline $\begin{array}{l}\text { Maximum } \\
\text { interruption/custome } \\
\mathrm{r}\end{array}$ & 10 & 50 & 100 \\
\hline $\begin{array}{l}\text { Estimated relative } \\
\text { cost }\end{array}$ & 20 & 50 & 100 \\
\hline
\end{tabular}

Table: 1 (Three level input taken for Taguchi analysis)

\begin{tabular}{|c|c|c|c|c|c|}
\hline A & B & $\mathrm{C}$ & $\mathrm{D}$ & $E$ & Yield \\
\hline 1 & 1 & 1 & 1 & 1 & 135 \\
\hline 1 & 1 & 1 & 1 & 2 & 165 \\
\hline 1 & 1 & 1 & 1 & 3 & 215 \\
\hline 1 & 2 & 2 & 2 & 1 & 276 \\
\hline 1 & 2 & 2 & 2 & 2 & 306 \\
\hline 1 & 2 & 2 & 2 & 3 & 356 \\
\hline 1 & 3 & 3 & 3 & 1 & 427 \\
\hline 1 & 3 & 3 & 3 & 2 & 457 \\
\hline 1 & 3 & 3 & 3 & 3 & 507 \\
\hline 2 & 1 & 2 & 3 & 1 & 330 \\
\hline 2 & 1 & 2 & 3 & 2 & 360 \\
\hline 2 & 1 & 2 & 3 & 3 & 410 \\
\hline 2 & 2 & 3 & 1 & 1 & 341 \\
\hline 2 & 2 & 3 & 1 & 2 & 371 \\
\hline 2 & 2 & 3 & 1 & 3 & 421 \\
\hline 2 & 3 & 1 & 2 & 1 & 182 \\
\hline 2 & 3 & 1 & 2 & 2 & 212 \\
\hline 2 & 3 & 1 & 2 & 3 & 262 \\
\hline 3 & 1 & 3 & 2 & 1 & 390 \\
\hline 3 & 1 & 3 & 2 & 2 & 420 \\
\hline 3 & 1 & 3 & 2 & 3 & 470 \\
\hline 3 & 2 & 1 & 3 & 1 & 241 \\
\hline 3 & 2 & 1 & 3 & 2 & 271 \\
\hline 3 & 2 & 1 & 3 & 3 & 321 \\
\hline 3 & 3 & 2 & 1 & 1 & 252 \\
\hline 3 & 3 & 2 & 1 & 2 & 282 \\
\hline 3 & 3 & 2 & 1 & 3 & 332 \\
\hline
\end{tabular}

Table: 2 (Result obtained after applying Taguchi three level design)
$\mathrm{A}=$ Miles of conductor
$\mathrm{B}=$ Feeder failures/yr.
$\mathrm{C}=$ Customer interruption/year
$\mathrm{D}=$ Maximum interruption/customer
$\mathrm{E}=$ Estimated relative cost
Yield = Output

Result of Taguchi analysis is as shown:

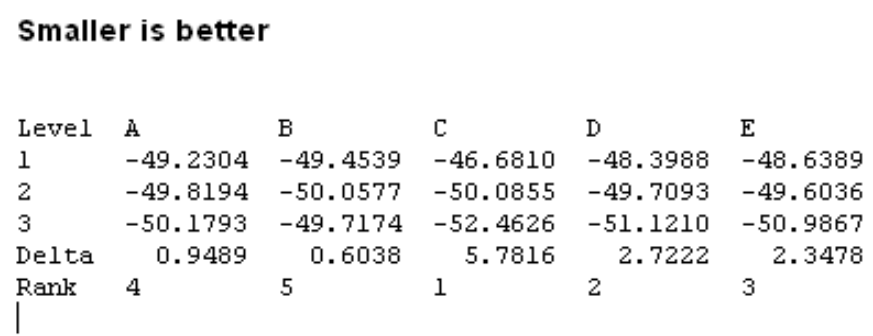

\section{Response Table for Means}

$\begin{array}{llllll}\text { Level } & \text { A } & \text { B } & \text { C } & \text { D } & \text { E } \\ 1 & 316 & 321.667 & 222.667 & 279.333 & 286 \\ 2 & 321 & 322.667 & 322.667 & 319.333 & 316 \\ 3 & 331 & 323.667 & 422.667 & 369.333 & 366 \\ \text { Delta } & 15 & 2.000 & 200.000 & 90.000 & 80 \\ \text { Rank } & 4 & 5 & 1 & 2 & 3\end{array}$

After doing the Taguchi analysis and getting the rank we get the factors according to priority basis as:

1. Customer interruption/year

2. Maximum interruption/customer

3. Estimated relative cost

4. Miles of conductor

5. Feeder failures/yr

Now applying AHP on the basis of the priority obtained by Taguchi analysis we have:

Applying AHP:

Determining weighing factors:

\begin{tabular}{|l|l|l|l|l|l|}
\hline & $\begin{array}{l}\text { Miles } \\
\text { of } \\
\text { conduct } \\
\text { or }\end{array}$ & $\begin{array}{l}\text { Feeder } \\
\text { failures } \\
\text { /yr }\end{array}$ & $\begin{array}{l}\text { Custom } \\
\text { er } \\
\text { interrup } \\
\text { tion/yea } \\
\text { r }\end{array}$ & $\begin{array}{l}\text { Maxim } \\
\text { um } \\
\text { interrup } \\
\text { tion/cus } \\
\text { tomer }\end{array}$ & $\begin{array}{l}\text { Estimat } \\
\text { ed } \\
\text { relative } \\
\text { cost }\end{array}$ \\
\hline $\begin{array}{l}\text { Miles } \\
\text { of } \\
\text { conduct } \\
\text { or }\end{array}$ & 1 & 3 & $1 / 7$ & $1 / 5$ & $1 / 3$ \\
\hline $\begin{array}{l}\text { Feeder } \\
\text { failures } \\
\text { /yr }\end{array}$ & $1 / 3$ & 1 & $1 / 9$ & $1 / 7$ & $1 / 5$ \\
\hline $\begin{array}{l}\text { Custom } \\
\text { er } \\
\text { interrup } \\
\text { tion/yea } \\
\text { r }\end{array}$ & 7 & 9 & 1 & 3 & 5 \\
\hline $\begin{array}{l}\text { Maxim } \\
\text { um } \\
\text { interrup } \\
\text { tion/cus } \\
\text { tomer }\end{array}$ & 5 & 7 & $1 / 3$ & 1 & 3 \\
\hline $\begin{array}{l}\text { Estimat } \\
\text { ed } \\
\text { relative } \\
\text { cost }\end{array}$ & 3 & 5 & $1 / 5$ & $1 / 3$ & 1 \\
\hline
\end{tabular}

Normalization matrix for criteria:

\begin{tabular}{|c|c|c|c|c|c|}
\hline & $\begin{array}{l}\text { Miles } \\
\text { of } \\
\text { condu } \\
\text { ctor }\end{array}$ & $\begin{array}{l}\text { Feede } \\
\mathrm{r} \\
\text { failur } \\
\text { es/yr }\end{array}$ & $\begin{array}{l}\text { Custo } \\
\text { mer } \\
\text { interr } \\
\text { uptio } \\
\text { n/year }\end{array}$ & $\begin{array}{l}\text { Maxi } \\
\text { mum } \\
\text { interr } \\
\text { uptio } \\
\text { n/cust }\end{array}$ & $\begin{array}{l}\text { Estim } \\
\text { ated } \\
\text { relati } \\
\text { ve } \\
\text { cost }\end{array}$ \\
\hline
\end{tabular}




\begin{tabular}{|l|l|l|l|l|l|}
\hline $\begin{array}{l}\text { Miles } \\
\text { of } \\
\text { condu } \\
\text { ctor }\end{array}$ & 0.06 & 0.12 & 0.08 & 0.04 & 0.03 \\
\hline $\begin{array}{l}\text { Feede } \\
\text { r } \\
\text { failur } \\
\text { es/yr }\end{array}$ & 0.02 & 0.04 & 0.06 & 0.03 & 0.02 \\
\hline $\begin{array}{l}\text { Custo } \\
\text { mer } \\
\text { interr } \\
\text { uptio } \\
\text { n/year }\end{array}$ & 0.43 & 0.36 & 0.56 & 0.64 & 0.52 \\
\hline $\begin{array}{l}\text { Maxi } \\
\text { mum } \\
\text { interr } \\
\text { uptio } \\
\text { n/cust } \\
\text { omer }\end{array}$ & 0.31 & 0.28 & 0.19 & 0.21 & 0.31 \\
\hline $\begin{array}{l}\text { Estim } \\
\text { ated } \\
\text { relati } \\
\text { ve } \\
\text { cost }\end{array}$ & 0.18 & 0.2 & 0.11 & 0.07 & 0.10 \\
\hline
\end{tabular}

Priority vector for criteria:

Miles of conductor $=(0.06+0.12+0.08+0.04+$ $0.03) / 5=0.066$

Feeder failures/yr. $=(0.02+0.04+0.06+0.03+$ $0.02) / 5=0.034$

Customer interruption/yr. $=(0.43+0.36+0.56+0.64+$ $0.52) / 5=0.502$

Maximum interruption/customer $=(0.31+0.28+0.19+0.21$ $+0.31) / 5=0.26$

Estimated relative cost $=(0.18+0.2+0.11+0.07+$ $0.10) / 5=0.132$

After we have obtained the priority of the factors and their respective weights we proceed for distribution planning using the Technique of Order Preference by Similarity to Ideal Solution (TOPSIS). In this method two artificial alternatives are hypothesized:

$\ddot{y}$ Ideal alternative: the one which has the best level for all attributes considered.

$\ddot{y}$ Negative ideal alternative: the one which has the worst attribute values.

TOPSIS selects the alternative that is the closest to the ideal solution and farthest from negative ideal alternative.

In the problem stated above let us first proceed to find the conductor layout from substation2. First to determine as to where to connect node 1 we proceed by constructing the normalized decision matrix.

Normalized decision matrix

\begin{tabular}{|l|l|l|l|l|l|}
\hline & $\begin{array}{l}\text { miles } \\
\text { of } \\
\text { condu } \\
\text { ctor }\end{array}$ & $\begin{array}{l}\text { estim } \\
\text { ated } \\
\text { cost }\end{array}$ & $\begin{array}{l}\text { feeder } \\
\text { failur } \\
\text { e }\end{array}$ & $\begin{array}{l}\text { custo } \\
\text { mer } \\
\text { interr } \\
\text { uptio } \\
\text { n }\end{array}$ & $\begin{array}{l}\text { maxi } \\
\text { mum } \\
\text { interr } \\
\text { uptio } \\
\text { n }\end{array}$ \\
\hline S2 & 5 & 5 & 3 & 3 & 3 \\
\hline
\end{tabular}

\begin{tabular}{|l|l|l|l|l|l|}
\hline 2 & 7 & 7 & 4 & 4 & 3 \\
\hline 3 & 13 & 13 & 10 & 10 & 6 \\
\hline 4 & 6 & 6 & 3 & 3 & 4 \\
\hline 13 & 8 & 8 & 4 & 4 & 5 \\
\hline
\end{tabular}

Weighted normalized decision matrix

\begin{tabular}{|l|l|l|l|l|l|}
\hline & $\begin{array}{l}\text { miles of } \\
\text { conductor }\end{array}$ & $\begin{array}{l}\text { estimat } \\
\text { ed cost }\end{array}$ & $\begin{array}{l}\text { feeder } \\
\text { failure }\end{array}$ & $\begin{array}{l}\text { custom } \\
\text { er } \\
\text { interrup } \\
\text { tion }\end{array}$ & $\begin{array}{l}\text { maxim } \\
\text { um } \\
\text { interrup } \\
\text { tion }\end{array}$ \\
\hline S2 & $\begin{array}{l}0.017818 \\
325\end{array}$ & $\begin{array}{l}0.0356 \\
3665\end{array}$ & $\begin{array}{l}0.0083 \\
282\end{array}$ & $\begin{array}{l}0.1229 \\
64385\end{array}$ & $\begin{array}{l}0.0800 \\
26311\end{array}$ \\
\hline 2 & 0.024945 & 0.0498 & 0.0111 & 0.1639 & 0.0800 \\
& 655 & 9131 & 043 & 52513 & 26311 \\
\hline 3 & 0.046327 & 0.0926 & 0.0277 & 0.4098 & 0.1600 \\
& 645 & 5529 & 608 & 81284 & 52623 \\
\hline 4 & 0.021381 & 0.0427 & 0.0083 & 0.1229 & 0.1067 \\
& 99 & 6398 & 282 & 64385 & 01749 \\
\hline 13 & 0.028509 & 0.0570 & 0.0111 & 0.1639 & 0.1333 \\
& 32 & 1864 & 043 & 52513 & 77186 \\
\hline
\end{tabular}

The above matrix is formed by considering the Priority vector for various criteria that were obtained as their weights.

The set of ideal solution $\mathrm{A} *$

\begin{tabular}{|l|l|l|l|l|}
\hline 0.0178183 & 0.035636 & 0.008328 & 0.1229643 & 0.080026 \\
25 & 65 & 265 & 85 & 311 \\
\hline
\end{tabular}

The set of negative ideal solution $\mathrm{A}^{\text {' }}$

\begin{tabular}{|l|l|l|l|l|}
\hline 0.0463276 & 0.0926552 & 0.0277608 & 0.40988 & 0.1600 \\
45 & 91 & 84 & 1284 & 52623 \\
\hline
\end{tabular}

Separation from ideal alternatives $\mathrm{S}_{\mathrm{i}}$ *

\begin{tabular}{|l|l|l|l|l|}
\hline 0 & 0.0440650 & 0.3052327 & 0.027840 & 0.071453 \\
& 37 & 81 & 214 & 034 \\
\hline
\end{tabular}

Separation from negative ideal alternative $\mathrm{S}_{\mathrm{i}}$ '

\begin{tabular}{|l|l|l|l|l|}
\hline 0.3052327 & 0.2635309 & 0 & 0.2977527 & 0.251112 \\
81 & 43 & & 12 & 41 \\
\hline
\end{tabular}

Relative closeness to ideal solution $\mathrm{C}_{\mathrm{i}}{ }^{*}$

\begin{tabular}{|c|l|}
\hline & $\mathrm{C}_{\mathrm{i}}{ }^{*}$ \\
\hline $\mathrm{S} 2$ & 1 \\
\hline 2 & 0.856744 \\
\hline 3 & 0 \\
\hline 4 & 0.914494 \\
\hline 13 & 0.778485 \\
\hline
\end{tabular}

Thus node 1 gets connected to substation 2

Second to determine as to where to connect node 2 we proceed by constructing the normalized decision matrix.

Normalized decision matrix

\begin{tabular}{|l|l|l|l|l|l|}
\hline & $\begin{array}{l}\text { miles } \\
\text { of } \\
\text { condu } \\
\text { ctor }\end{array}$ & $\begin{array}{l}\text { estim } \\
\text { ated } \\
\text { cost }\end{array}$ & $\begin{array}{l}\text { feeder } \\
\text { failur } \\
\text { e }\end{array}$ & $\begin{array}{l}\text { custo } \\
\text { mer } \\
\text { interr } \\
\text { uptio } \\
\text { n }\end{array}$ & $\begin{array}{l}\text { maxi } \\
\text { mum } \\
\text { interr } \\
\text { uptio } \\
\text { n }\end{array}$ \\
\hline S2 & 5 & 5 & 3 & 3 & 3 \\
\hline
\end{tabular}




\begin{tabular}{|l|l|l|l|l|l|}
\hline 1 & 7 & 7 & 4 & 4 & 3 \\
\hline 3 & 5 & 5 & 3 & 3 & 4 \\
\hline 4 & 16 & 16 & 12 & 12 & 8 \\
\hline 13 & 7 & 7 & 4 & 4 & 5 \\
\hline
\end{tabular}

Weighted normalized decision matrix

\begin{tabular}{|l|l|l|l|l|l|}
\hline & $\begin{array}{l}\text { miles } \\
\text { of } \\
\text { condu } \\
\text { ctor }\end{array}$ & $\begin{array}{l}\text { estim } \\
\text { ated } \\
\text { cost }\end{array}$ & $\begin{array}{l}\text { feeder } \\
\text { failur } \\
\text { e }\end{array}$ & $\begin{array}{l}\text { custo } \\
\text { mer } \\
\text { interr } \\
\text { uptio } \\
\text { n }\end{array}$ & $\begin{array}{l}\text { maxi } \\
\text { mum } \\
\text { interr } \\
\text { uptio } \\
\text { n }\end{array}$ \\
\hline S2 & 0.016 & 0.032 & 0.007 & 0.108 & 0.070 \\
& 41811 & 83622 & 32317 & 12449 & 33023 \\
& 4 & 7 & 3 & 9 & 2 \\
\hline 1 & 0.022 & 0.045 & 0.009 & 0.144 & 0.070 \\
& 98535 & 97071 & 76423 & 16599 & 33023 \\
& 9 & 8 & 1 & 8 & 2 \\
\hline 3 & 0.016 & 0.032 & 0.007 & 0.108 & 0.093 \\
& 41811 & 83622 & 32317 & 12449 & 77364 \\
& 4 & 7 & 3 & 9 & 2 \\
\hline 4 & 0.052 & 0.105 & 0.029 & 0.432 & 0.187 \\
& 53796 & 07592 & 29269 & 49799 & 54728 \\
& 4 & 7 & 3 & 5 & 4 \\
\hline 13 & 0.022 & 0.045 & 0.009 & 0.144 & 0.117 \\
& 98535 & 97071 & 76423 & 16599 & 21705 \\
& 9 & 8 & 1 & 8 & 3 \\
\hline
\end{tabular}

The above matrix is formed by considering the Priority vector for various criteria that were obtained as their weights.

The set of ideal solution $\mathrm{A}^{*}$

\begin{tabular}{|l|l|l|l|l|}
\hline 0.016418 & 0.032836 & 0.007323 & 0.108124 & 0.070330 \\
114 & 227 & 173 & 499 & 232 \\
\hline
\end{tabular}

The set of negative ideal solution A'

\begin{tabular}{|l|l|l|l|l|}
\hline 0.052537 & 0.105075 & 0.029292 & 0.432497 & 0.187547 \\
964 & 927 & 693 & 995 & 284 \\
\hline
\end{tabular}

Separation from ideal alternatives $\mathrm{S}_{\mathrm{i}} *$

\begin{tabular}{|r|r|r|r|r|}
\hline & 0.038994 & 0.023443 & 0.354913 & 0.060983 \\
0 & 769 & 411 & 905 & 326 \\
\hline
\end{tabular}

Separation from negative ideal alternative $\mathrm{S}_{\mathrm{i}}$,

\begin{tabular}{|l|l|l|l|l|}
\hline 0.354913 & 0.318784 & 0.347875 & 0 & 0.304679 \\
905 & 129 & 752 & & 872 \\
\hline
\end{tabular}

Relative closeness to ideal solution $\mathrm{C}_{\mathrm{i}}{ }^{*}$

\begin{tabular}{|c|c|}
\hline & $\mathrm{C}_{\mathrm{i}}^{*}$ \\
\hline $\mathrm{S} 2$ & 1 \\
\hline 1 & 0.891009 \\
\hline 3 & 0.936865 \\
\hline 4 & 0 \\
\hline 13 & 0.833225 \\
\hline
\end{tabular}

Thus node 2 gets connected to substation 2

Proceeding in the manner stated above we get the conductor layout as shown in fig 2.

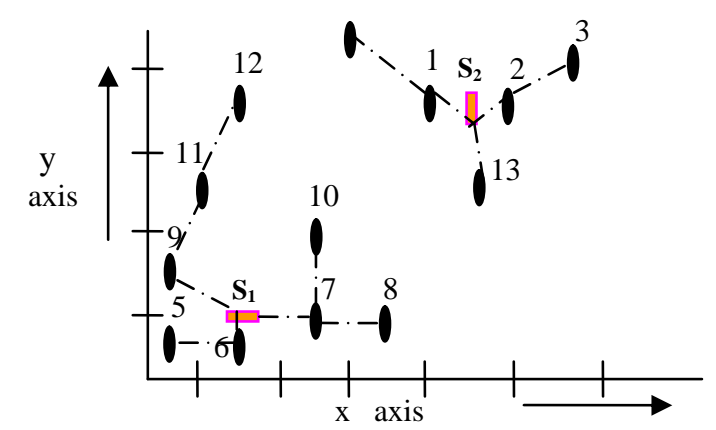

Figure 2 (Showing the final conductor layout from the substations)

\section{CONCLUSION}

Experts rating on various factors such as

i). Miles of conductor.

ii). Feeder failures/year.

iii). Customer interruption/year.

iv). Maximum interruption/customer.

v). Estimated relative cost.

are to be considered before deciding the conductor layout in distribution planning system. Conventional techniques for suggestion of conductor layout do not take into consideration such factors and decisions of experts. In conductor layout using Analytical Hierarchy Process reliability aspects have been taken care of but the priority of these factors have been decided in random. No technique has been used to determine the priority of importance of these factors. In this paper a model has been proposed where a systematic solution process has been used to determine the priority of these factors then an Analytical Hierarchy Process has been applied for finding the weight of these factors and ultimately distribution planning has been carried out by Technique of Order Preference by Similarity to Ideal Solution. Thus we can claim the suggested method to be more robust and closer to practical than the existing ones.

\section{REFERENCES}

[1] Belgin Turkay and Taylan Artac. (2005), "Optimal Distribution Network Design Using Genetic Algorithm," Electric Power Components and Systems, 33; 513-524.

[2] J.F.Gomez et al. (2004), "Ant Colony System Algorithm for the Planning of Primary Distribution Circuits," IEEE Transactions on Power Systems, Vol. 19, No. 2.

[3] K.K.Li and T.S.Chung. (2004), "Distribution Planning Using Rule Based Expert System Approach," IEEE International Conference on Electric Utility Deregulation and Power Technologies(DRPT 2004).

[4] Eloy Diaz-Dorado et al. (2002), "Application of Evolutionary Algorithms for the Planning of Urban Distribution Networks of Medium Voltage," IEEE Transaction Power System, Vol. 17, No. 3.

[5] Sujit Mondal and Anil Pahwa. (2002), "Optimal Selection ofConductors for Distribution Feeders," IEEE Transactions on Power Systems, Vol. 17, No. 1.

[6] D.L.Wall et al. (1979), "An Optimization Model for Planning Radial Distribution Networks," IEEE Transactions on Power Apparatus and Systems, Vol..PAS-98, No. 3.

[7] W.M. Lin et al. (1997), "A new Approach for Distribution Feeder Reconfiguration for Loss Reduction and Service Restoration," IEEE PES.

[8] Dale M.Crawford and Stewart B. Holt. (1975), "A Mathematical Optimization Technique For Locating Sizing Distribution Substations, and Driving Their Optimal Service Areas," IEEE. Trans. on Power Apparatus and Systems, Vol. PAS 94, No. 2, pp. 230-235. 
[9] M.A. El-Kady. (1984), “Computer Aided planning of Distribution Substation and Primary Feeders," IEEE. Trans. on Power Apparatus and Systems, Vol. PAS 103, No. 6, pp. 1183-1189.

[10] T.Gonen, I.J. Ramirez-Rosado. (1987), "Optimal Multi Stage Planning of Power Distribution Systems,” IEEE Trans. on Power Delivery,Vol. PWRD-2, No.2, pp 512-519.

[11] J.Partanen. (1990), "A Modified Dynamic Programming Algorithm for Sizing, Locating and Timing of Feeder Reinforcements," IEEE Trans. on Power Delivery, Vol.5, No.1, pp 227-283.

[12] V.Mirinda, J.V.Ranito, L.M. Proenca. (1994), "Genetic Algorithm in Optimal Multistage Distribution Network Planning”, IEEE Trans. On Power Systems, vol. 9, No. 4, pp. 1927-1933.

[13] S.Chakravorty and M.Thukral . (2007), "Optimal Allocation of Load Using Optimization Technique", International conference CISSE

[14] Robert H.Fletcher. (2007), "Optimal Distribution System Horizon Planning: Application,” IEEE Trans. Power Syst., Vol. 22.

[15] S.K.Khator and L.C. Leung. (1997), "Power Distribution Planning: A review of models and issues," IEEE Trans. Power Syst., vol.12, pp 1151-1159.

[16] J.L.Bernal-Agustin.(1998), “ Aplicacion de Algoritmos Geneticos al Diseno Optimo de Sistemas de Distribucion de Energia Electrica," Ph.D. dieesrtation, University de Zaragoza, Espana.

[17] J.T.Boardman and C.C. Meekiff. (1985), "A branch and bound formulation of an electricity distribution planning problem," IEEE Trans. Power App. Syst., vol. 104, pp. 2112-2118.

[18] K.Nara et al. (1992), "Distribution system expansion planning b multistage branch exchange," IEEE Trans. Power syst., vol. 7, pp.208-214.

[19] B.Turkay and T.Artac. (2005), "Optimal distribution network design using genetic algorithm," Taylor \& Francis Electric Power Components and Systems, 33. pp. 513-524.

[20] Z. Sumic et al. (1993), "Automated underground residential distribution Part 2: Prototype implementation and results," IEEE Trans. Power Delivery, vol. 8, pp. 644-650.

[21] E.C. Yeh, S.S. Venkata, and Z. Sumic. (1996), "Improved distribution system planning using computational evolution,” IEEE Trans. Power Syst., vol. 11, pp. 668-674.

[22] V.Miranda, J.V.Raito, and L.M.Proenca. (1994), "Genetic algorithm in optimal multistage distribution network planning," IEEE Trans. Power Syst., vol.9, pp.1927-1933.

[23] I.J.Ramirez-Rosado and J.L.Bernal Agustin. (1995), "Optimization of power distribution network design by application of genetic algorithm,” Int.J.Power Energy Syst., vol.15, no.3, pp.104-110.

[24] Ramirez Rosado, I.J., Bernal Agustin, J.L. (1998), “Genetic algorithm applied to design of large power distribution system,” IEEE Trans. Power Syst., vol.13, pp.696-703.

[25] P.M.S.Carvalho and L.A.F.M. Ferreira. (1998), "Optimal distribution network expansion planning under uncertainty by evolutionary decision convergence,” Int.J.Elect. Power Energy Syst., vol. 20, no. 2, pp. 125-129.

[26] M.Dorigo, V.Maniezzo and A.Coloni. (1996), "The ant system: Optimization by a colony of cooperating agents," IEEE Trans. Syst. Man, Cybern.B, vol. 26, pp. 29-41.

[27] M.Dorigo amd L.M.Gambardella. (1997), “Ant colony system: A cooperative learning approach to the traveling salesman problem,"IEEE Trans. Evol.. Comput., vol. 1, pp. 29-41.

[28] Y.Da Silva, J.Di Girolano and A.Ferreira. (1999), "Optimizacion de circuitos aereos de distribucion en campos petroleros" (in Spanish), in Proc. IEEE Andean Region Conf., vol. II, 1, pp. 967-972.

[29] C.E.Advirson. (1940), "Diversified demand method of estimation residential distribution transformer load." Edison Elect. Inst Bulletin,vol. 8, pp. 469-479.

[30] A.J.Urdaneta and V.Chankong. (1989), "A multiple objective minimax approach for controller setting of systems running under disturbances," in Control: Theoryand Advanced Technology, Invited Paper Special Issue on Multiple Objective, Discrete, Dynamic Systems. Tokyo, Japan: MITA,vol.5.

[31] E.Diaz Dorado, J.Cidras and E. Miguez. (2002), "Application of evolutionary algorithms for the planning of urban distribution networks of medium voltage," IEEE Trans. Power Syst., vol. 17, pp. 879-884.

[32] Sandeep Chakravorty and Manish thukral. (2009), "Choosing Distribution Sub Station Location Using Soft Computing Technique”, International Conference on Advances in Computing, Communication and Control - 2009 Mumbai. India.

[33] Sandeep Chakravorty and Manish Thukral. (2008), "Using Genetic Algorithm to Determine the Optimum Location of the Sub Station", International Workshop and IEEE EDS Mini Colloquia on Nanometer CMOS Technology (WIMNACT 2008) on Nanoelectronics 6-8 March 2008.
[34] Sandeep Chakravorty, Smarajit Ghosh, (2009), “An Improvised Method for Distribution of Loads and Configuration of Distribution Sub Station", International Journal of Engineering Research and Industrial Applications. Vol.2 No. II, pp. 269-280. 2009.

[35] Sandeep Chakravorty, Smarajit Ghosh, "Fuzzy Based Distribution Planning Technique", Journal of Electrical Engineering. Volume 9 2009 - Edition: 2, pp. 38-43

[36] Sandeep Chakravorty, Smarajit Ghosh, (2009), "Distribution Planning Based on Reliability and Contingency Criteria", International Journal of Computer and Electrical Engineering. Vol.1 No. 2, pp. 156-161. 2009.

[37] Sandeep Chakravorty, Smarajit Ghosh, (2009), "A Novel Approach to Distribution Planning in an Unstructured Environment”, International Journal of Computer and Electrical Engineering. Accepted for publication

[38] Sandeep Chakravorty, Smarajit Ghosh, (2009), "A Hybrid Model of Distribution Planning", International Journal of Computer and Electrical Engineering. Accepted for publication.

Sandeep chakravorty serving as a Reader in Sikkim Manipal Institute of Technology, Department of Electrical and Electronics Engineering under Sikkim Manipal University. He did his B.E. from Sikkim Manipal Institute of Technology, Sikkim in Electrical \& Electronics Engineering and M.E. in Software Engineering from Birla Institute of Technology, Mesra, Ranchi. India. Currently he is doing his Ph.D. in Sikkim Manipal University.

Smarajit Ghosh serving as a Professor in Thapar University, Department of Electrical and Instrumentation Engineering. He did his B.Tech., M.Tech. in Electrical Machines and Power Systems from Calcutta University in 1994 and 1996 respectively. Finally, he did his Ph.D. from Indian Institute of Technology, Khapargpur, India in 2000. His research areas include load-flow study, network reconfigurations, optimum capacitor allocation, application of soft computing in Elctrical Power Distribution Systems. 